\title{
Effects of Long-Term Supplementation of Blue-Green Algae on Lipid Metabolism in C57BL/6J mice
}

\author{
Yue Yang, Bohkyung Kim, Young-Ki Park, and Ji-Young Lee* \\ Department of Nutritional Sciences, University of Connecticut, USA
}

Received: October 27, 2013; Accepted: January 23, 2014, Published: January 27, 2014

*Corresponding author: Ji-Young Lee, Department of Nutritional Sciences, University of Connecticut, Storrs, Connecticut 06269, USA, Tel: 860486-1827; Fax: 860-486-3674; E-mail: ji-young.lee@uconn.edu

\begin{abstract}
Dyslipidemia is a primary risk factor for cardiovascular disease. In this study, we investigated the effect of long-term supplementation of two blue-green algae (BGA) species, i.e., Nostoc commune var. sphaeroides Kützing (NO) and Spirulina platensis (SP), on lipid metabolism in vivo. Male C57BL/6J mice were fed an AIN-93G/M diet supplemented with 2.5 or $5 \%$ (wt/wt) NO or SP for 6 months. Mice fed NO and SP showed lower plasma total cholesterol (TC) and triglyceride (TG) concentrations than control at certain months during 6 month experimental period. Both BGA supplementation for 6 months significantly increased hepatic TC contents whereas SP-fed groups had significantly less TG levels in the liver compared with control and NO groups. None of BGA-fed animals showed significantly different mRNA levels of sterol regulatory element binding protein 2, while 3-hydroxy3-methylglutaryl coenzyme A reductase and low-density lipoprotein receptor (LDLR) expression was higher in NO groups than the other groups in the liver. Furthermore, NO supplementation increased the hepatic expression of acetyl-CoA carboxylase 1 , stearoyl CoA desaturase 1 , carnitine palmitoyltransferase $1 \alpha$, and acyl-CoA oxidase 1 but SP did not elicit any significant changes in mRNA levels of the genes compared with control. LDLR protein level was significantly higher in NO 2.5\% and SP 5\%, as compared to the control and NO 5\% groups; while the level of fatty acid synthase protein in the liver was significantly higher in NO $5 \%$ and SP 5\%, than that in the control group. In conclusion, our results suggest that long-term supplementation of NO and SP decreased plasma TC and TG concentrations. Therefore, supplementation of NO and SP may be potentially beneficial for preventing dyslipidemia-associated chronic diseases.
\end{abstract}

Keywords: Blue-green algae; Cholesterol; Nostoc commune var. sphaeroides Kützing; Spirulina platensis; Triglyceride

\section{Abbreviations}

ACOX-1: Acyl-CoA 0xidase 1; BGA: Blue Green Algae; CPT-1 $\alpha$ : Carnitine palmitoyltransferase $1 \alpha$; CVD: Cardiovascular Disease; FAS: Fatty Acid Synthase; GAPDH : Glyceraldehyde 3-phosphate dehydrogenase; HMGR : 3-hydroxy-3-methylglutaryl coenzyme A reductase; INSIGs: Insulin-Induced Genes; LDL: Low-Density Lipoprotein; LDL-C: LDL Cholesterol; LDLR: LDL Receptor; NAFLD: Non-Alcoholic Fatty Liver Disease; NO: Nostoc commune var. sphaeroides Kützing; qRT-PCR: Quantitative Real Time PCR; SCAP: SREBP Cleavage Activating Protein; SCD-1: Stearoyl Co-A desaturase 1; SREBP: Sterol Regulatory Element Binding Protein;
SP: Spirulina platensis; TC: Total Cholesterol; TG: Triglyceride

\section{Introduction}

Cardiovascular disease (CVD) is the leading cause of death in the developed countries [1]. Atherosclerosis, a hallmark of CVD, is a chronic process characterized by accumulation of fatty plaques in aorta and coronary arteries [1]. The progression of atherosclerosis is accelerated by dyslipidemia, inflammation, hypertension, obesity and smoking [2]. Statins, the mostly prescribed cholesterol-lowering drugs, have been successfully used to lower plasma total and low-density lipoprotein (LDL) cholesterol concentrations for the prevention of CVD. However, due to known side effects of statins such as myopathy $[3,4]$, natural products that have hypocholesterolemic effects with no adverse side effects may serve as a good alternative for lowering CVD risk.

Blue-green algae (BGA) are one of the most primitive life forms on earth and are thought to contribute significantly to the global ecology [5]. BGA are rich in essential amino acids, minerals, fibers, carotenoids and other bioactive components [6-9]. Edible BGA have been utilized as food and herbal medicine in Asian, African and South American countries for many centuries $[10,11]$. Studies have shown hypolipidemic and anti-inflammatory effects of several BGA species [12]. In both mice and humans, Spirulina plantensis (SP) has been shown to lower plasma total cholesterol (TC) and LDL cholesterol (LDL-C), and triglyceride (TG) concentrations $[13,14]$. Nostoc commune var. sphaeroides Kützing (NO), another BGA species has also been used in indigenous countries to treat inflammation, night blindness, indigestion, and chronic fatigue [15-17]. Furthermore, in male Wistar rats that were fed a high cholesterol diet, 5\% NO supplementation elicited a hypolipidemic effect [18]. Our previous study also found that male C57BL/6J mice fed a 5\% NO-supplemented diet for 4 weeks showed significantly lower plasma TC and TG levels [19]. We also demonstrated that lipid extract from NO inhibited the maturation of sterol regulatory element binding protein 1c (SREBP-1c) and SREBP-2, consequently repressing the expression of lipogenic genes and cholesterol biosynthetic and uptake genes, respectively, in HepG2 cells [20].

Although we and others have suggested NO and SP may have an athero-protective property by improving healthy plasma 
lipid profiles, the effects of long-term consumption of BGA on cholesterol and lipid metabolism have not been studied. The objective of this study was to investigate whether long-term consumption of NO and SP can lower plasma lipid levels in mice. Male C57BL/6J mice were fed an experimental diet supplemented with $2.5 \%$ or $5 \%$ of NO or SP for 6 months from the age of 4 weeks and plasma and liver lipid profiles and hepatic gene expression related to lipid metabolism were studied.

\section{Materials and Methods}

\section{Diet and animal study}

Male C57BL/6J mice at 4 weeks of age were purchased from Jackson Laboratory (Bar Harbor, ME) and randomly assigned to one of five groups, i.e., control, $2.5 \%$ or $5 \%$ of NO or SP. Mice were housed in a polycarbonate cage under a 12-h light/dark cycle. Powdered NO and SP were generously provided by Algaen Corp (Winston-Salem, NC) and Earthrise Nutritionals (Irvine, CA), respectively. Mice were fed AIN-93G/M diets supplemented with BGA for 6 months and detailed diet composition can be found elsewhere [21]. Blood samples were obtained into tubes containing EDTA (BD Vacutainer) monthly by tail bleeding and terminal blood samples were drawn by cardiac puncture after 4 h-fasting under anesthesia with ketamine $\mathrm{HCl}$ (50 mg/Kg)/ xylazine $(10 \mathrm{mg} / \mathrm{Kg})$. Liver samples were snap frozen in liquid nitrogen and stored at $-80^{\circ} \mathrm{C}$ until use. All animal experiments were approved by the Institutional Animal Care and Use Committee at the University of Nebraska-Lincoln.

\section{Plasma chemistry}

Blood samples were centrifuged for $10 \mathrm{~min}$ at $1,500 \mathrm{xg}$ at $4^{\circ} \mathrm{C}$ to remove red blood cells and plasma TC and TG concentrations were measured using Cholestech LDX Lipid Profile Glucose cassettes by the Cholestech LDX System (Cholestech Corporation, Hayward, CA).

\section{Gene expression analysis by quantitative realtime PCR (qRT-PCR)}

qRT-PCR analysis for gene expression was conducted as previously described using the SYBR Green procedure and CFX96 ${ }^{\mathrm{TM}}$ realtime PCR detection system (BioRad, Hercules, CA)
$[20,22]$. Primer sequences were designed according to GenBank database using the Beacon Designer software (Premier Biosoft, Palo Alto, CA) and the list of primer sequences is provided in Table 1. Glyceraldehyde 3-phosphate dehydrogenase (GAPDH) was used as an internal control to normalize data.

\section{Western blot analysis}

Protein from liver samples was extracted and Western blot analysis was performed as previously described [15] using antibodies against fatty acid synthase (FAS; Santa Cruz Biotechnology, Santa Cruz, CA), LDL receptor (LDLR; Abcam, Cambridge, MA), SREBP-2 (Santa Cruz Biotechnology, Santa Cruz, CA) and $\beta$-actin (Sigma, St. Louis, MO). $\beta$-Actin was used as a loading control.

\section{Statistical analysis}

One-way analysis of variance and Newman-Keuls post hoc test were performed to identify significant differences between groups using Graph Pad Prism 5 (GraphPad Software, Inc., La Jolla, CA). P values of $<0.05$ were considered statistically significant. Data are expressed as means \pm standard error mean.

\section{Results}

\section{Effect of BGA supplementation on plasma lipids}

The effect of NO and SP supplementation on plasma TC and TG concentrations were measured monthly throughout 6 months. Due to insufficient amount of plasma samples, we were not able to measure TC levels at 2 month and TG levels at 5 month of experimental period. NO supplementation at either $2.5 \%$ or $5 \%$ significantly lowered plasma TC levels from the first to the fifth month whereas $2.5 \%$ SP significantly decreased TC levels at 3 and 5 months compared with control (Figure 1A). Plasma TG concentrations were significantly lower in 5\% NO group at 2 month and in $2.5 \%$ NO group at 6 month, than in the control group (Figure 1B). We also observed decreased plasma TG levels in $2.5 \%$ SP-fed mice at 4 and 6 month after the BGA feeding.

\section{Effect of BGA supplementation on hepatic lipids}

Liver lipid content largely affects plasma TC and TG concentrations. Therefore, mice on the experimental diet containing

Table 1: Primer sequences for qPCR analysis.

\begin{tabular}{|l|r|r|}
\hline Gene & Forward Primer & Reverse Primer \\
\hline HMGR & 5'-GGGCCCCACATTCACTCTT-3' & 5'-TGGTGCCAACTCCAATCACA-3' \\
\hline LDLR & 5'-GGGTTGATTCCAAACTCCATTC-3' & 5'-TCCGATTGCCCCCATTG-3' \\
\hline SREBP-2 & 5'-GTGTGCGGAGGAGAAAATCC-3' & 5'-CCCATGGCCGCTGTCA-3' \\
\hline SREBP-1c & 5'-GGAGCCATGGATTGCACATT-3' & 5'-GGCCCGGGAAGTCACTGT-3' \\
\hline FAS & 5'-TCCTGGAACGAGAACACGATCT-3' & 5'-GAGACGTGTCACTCCTGGACTTG-3' \\
\hline ACC & 5'-CGCTCAGGTCACCAAAAAGAAT-3' & 5'-GTCCCGGCCACATAACTGAT-3' \\
\hline SCD-1 & 5'-CAGTGCCGCGCATCTCT-3' & 5'-CCCGGGATTGAATGTTCTTG-3' \\
\hline CPT1 & 5'-GAACCCCAACATCCCCAAAC-3' & 5'-TCCTGGCATTCTCCTGGAAT-3' \\
\hline ACOX & 5'-CCCAAGACCCAAGAGTTCATTC-3' & 5'-CAGGCCACCACTTGATGGA-3' \\
\hline GAPDH & 5'-TGTGTCCGTCGTGGATCTGA-3' & 5'-CCTGCTTCACCACCTTCTTGAT-3' \\
\hline
\end{tabular}


A
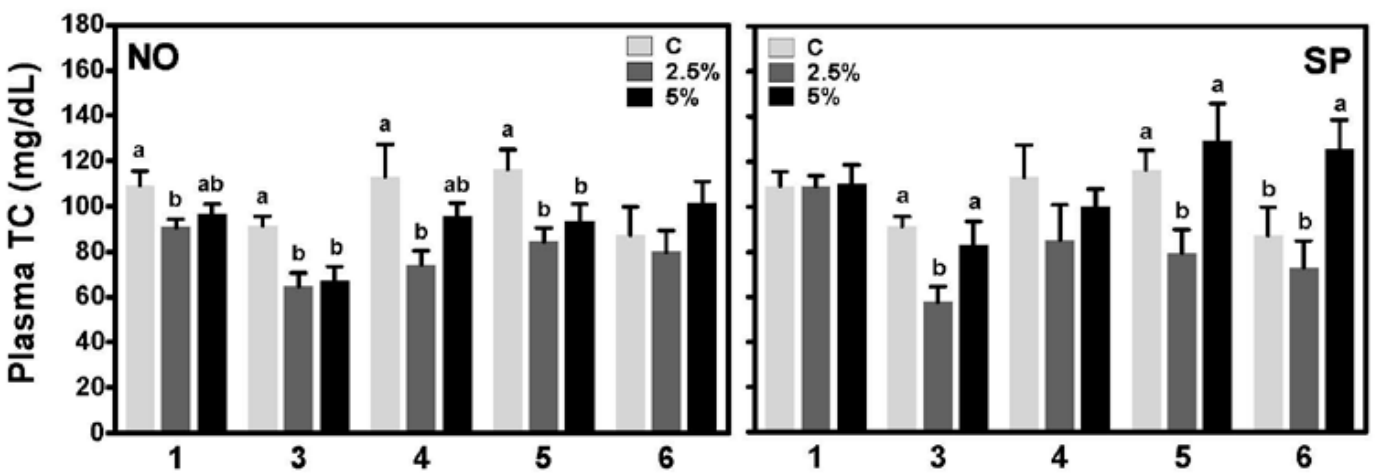

B

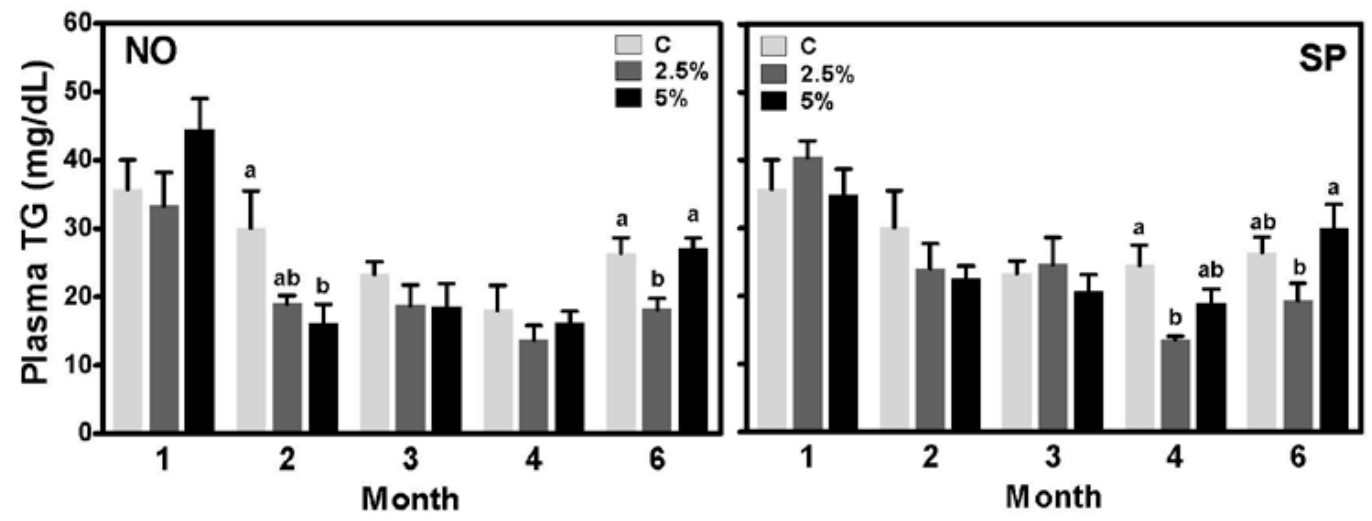

Figure 1: Plasma TC and TG concentrations of male C57BL/6J mice, which were fed an AIN-93G/M control or a BGA supplemented diet (2.5\% and 5\% for NO and SP, by weight). TC (A) and TG (B) concentrations were measured at intervals during 6 months. Data are shown as means \pm SEM. $\mathrm{n}=7-8$. Bars with a different letter are significantly different $(\mathrm{P}<0.05)$ within the same month.

A

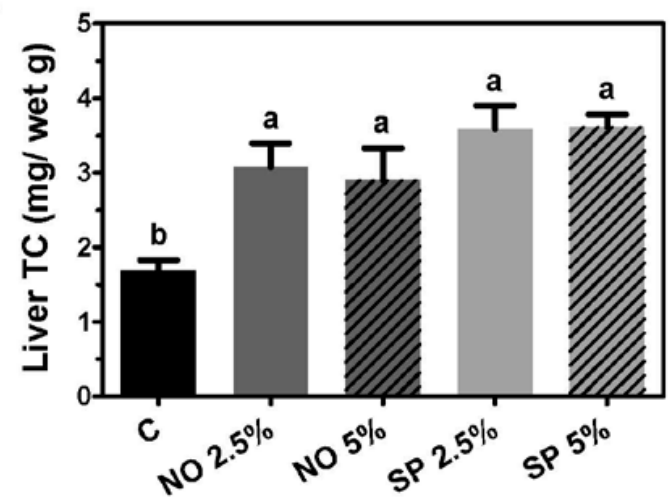

B

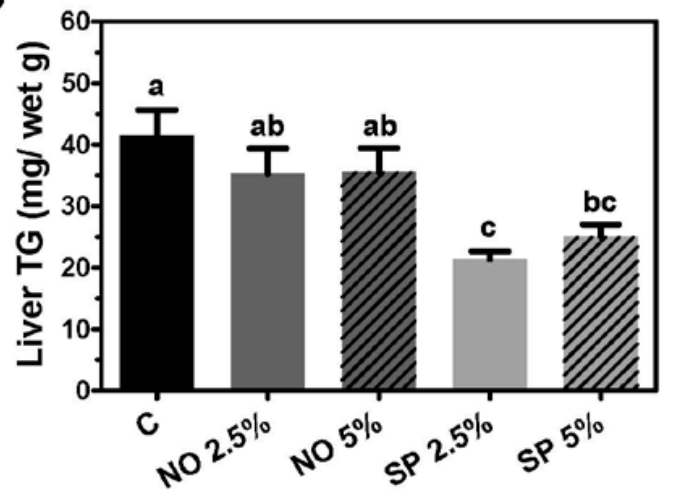

Figure 2: Lipid contents of the livers of male C57BL/6J mice, which were fed an AIN-93G/M control or a BGA supplemented diet (2.5\% and 5\% for NO and SP, by weight) for 6 months. Hepatic TC (A) and TG (B) levels were measured and expressed mg/g of wet weight. Data are shown as means \pm SEM. $n=7-8$. Bars with a different letter are significantly different $(\mathrm{P}<0.05)$.

BGA for 6 months were analyzed for their hepatic TC and TG content. Compared with control, all BGA-fed animals showed significantly higher TC levels in the liver (Figure 2A). Hepatic TG contents in NO $2.5 \%$ and $5 \%$-fed mice were not significantly different either from control or from SP-fed mice (Figure 2B). However, both $2.5 \%$ and $5 \%$ SP supplementation significantly decreased TG levels in the liver as compared to the control.
Alteration in hepatic expression of genes in cholesterol and lipid metabolism by BGA supplementation

To elucidate the mechanism underlying the lipid-lowering effect of BGA, the expression of the genes involved in the hepatic cholesterol and lipid metabolism was determined by qRT-PCR. There was no significant difference in SREBP-1c and SREBP-2 
mRNA levels between groups (Table 2). NO supplementation showed higher 3-hydroxy-3-methylglutaryl coenzyme A reductase (HMGR) and LDLR mRNA levels than control and SP-fed animals. Furthermore, lipogenic genes, e.g., acetyl-coA carboxylase 1 (ACC-1) and stearoyl-coA desaturase 1 (SCD1) were expressed higher in the livers of NO-fed mice than the other groups. Expression of carnitine palmitoyltransferase $1 \alpha$ (CPT-1 $\alpha$ ) and acyl-CoA oxidase 1 (ACOX-1), which are ratelimiting enzymes for mitochondrial and peroxisomal fatty acid $\beta$-oxidation, respectively, was significantly increased by both $2.5 \%$ and $5 \%$ NO supplementation as compared to the control.

Protein levels of mature SREBP-2 were not significantly different between groups (Table 3). LDLR protein levels in $2.5 \%$ NO and 5\% SP-fed animals were significantly higher than control and $5 \%$ NO groups. Both BGA at $2.5 \%$ did not significantly alter FAS protein levels whereas 5\% NO and SP elicited a significant increase in FAS than control.

\section{Discussion}

Various health benefits of BGA have been suggested including antioxidant, anti-inflammatory, as well as plasma cholesterol and TG-lowering properties [12]. However, concerns over safety and lack of scientific evidence for their health benefits have counteracted their use for human consumption. Previously we reported that two BGA species, i.e., NO and SP, did not contain detectable levels of microcystins, major algal toxins, and $5 \%$ supplementation of the BGA did not induce evident side-effects in mice during 6 months of supplementation [21]. In the current study, we aimed to evaluate effects of long-term supplementation of NO and SP on lipid metabolism in mice. This is the first study, to our knowledge, which demonstrates that BGA provide hypolipidemic effects as early as 1 month during 6 month feeding depending on type and supplemental level of BGA.

Dyslipidemia is a major risk factor for CVD [23] and the primary cause of death in the developed countries [24]. The TC- and TG-lowering effects of BGA have been previously demonstrated in animal studies as well as human clinical trials [14,25-27]. We also reported that C57BL/6] mice fed a 5\% NOsupplemented diet for 4 weeks showed decreased plasma TC and TG levels with concomitant increases in HMGR and LDLR expression, which was attributed to a decrease in intestinal cholesterol absorption [19]. In the present study, we also found that NO treatment lowered plasma TC concentrations throughout the intervention period, with the exception of 6 months. SP supplementation at $2.5 \%$, but not $5 \%$, also decreased plasma TC levels although the reduction was less evident and took longer to be effective than NO. Reason for the different efficacy in lowering plasma TC levels between NO and SP is unknown but it is likely due to their compositional difference. Compositional analysis and identification of bioactive compounds are necessary to understand the difference. Identification of bioactive compounds is currently underway in our laboratory.

After 6 month BGA supplementation, hepatic levels of TC, predominantly free cholesterol was significantly increased in both BGA-fed mice. In general, SREBP-2 induces the transcription of the genes encoding cholesterol biosynthetic enzymes and LDL uptake, such as HMGR and LDLR, when cellular cholesterol is low [28-32]. Transcriptional activity of SREBP-2 is primarily regulated at the posttranscriptional level involving insulin-induced genes (INSIGs) and SREBP cleavage-activating protein (SCAP). SCAP/SREBP precursor complex retains in

Table 2: Hepatic mRNA Abundance of Mice Fed an AIN93G/M Diet Supplemented with BGA for 6 Months.

\begin{tabular}{|l|c|c|c|c|}
\hline & Control & NO 2.5\% & NO 5\% & SP 2.5\% \\
\hline SREBP-2 & $1.2 \pm 0.1$ & $1.5 \pm 0.2$ & $1.5 \pm 0.2$ & $1.1 \pm 0.2$ \\
\hline LDLR & $1.5 \pm 0.2^{\mathrm{ab}}$ & $2.1 \pm 0.3^{\mathrm{a}}$ & $2.1 \pm 0.3^{\mathrm{a}}$ & $1.4 \pm 0.2^{\mathrm{ab}}$ \\
\hline HMGR & $1.3 \pm 0.2^{\mathrm{b}}$ & $2.7 \pm 0.5^{\mathrm{a}}$ & $1.9 \pm 0.3^{\mathrm{ab}}$ & $1.3 \pm 0.2^{\mathrm{b}}$ \\
\hline SREBP-1c & $1.5 \pm 0.2$ & $1.6 \pm 0.2$ & $1.4 \pm 0.2$ & $1.3 \pm 0.3$ \\
\hline FAS & $0.8 \pm 0.1$ & $1.1 \pm 0.2$ & $0.2^{\mathrm{b}}$ & $0.7 \pm 0.1$ \\
\hline ACC-1 & $0.9 \pm 0.1^{\mathrm{b}}$ & $1.6 \pm 0.3^{\mathrm{a}}$ & $1.4 \pm 0.1^{\mathrm{a}}$ & $1.1 \pm 0.1$ \\
\hline SCD-1 & $1.1 \pm 0.2^{\mathrm{b}}$ & $2.6 \pm 0.4^{\mathrm{a}}$ & $2.8 \pm 0.2^{\mathrm{a}}$ & $0.1^{\mathrm{b}}$ \\
\hline ACOX-1 & $1.2 \pm 0.1^{\mathrm{b}}$ & $2.8 \pm 0.6^{\mathrm{a}}$ & $2.5 \pm 0.2^{\mathrm{a}}$ & $1.8 \pm 0.2^{\mathrm{b}}$ \\
\hline CPT-1 $\alpha$ & $1.6 \pm 0.2^{\mathrm{b}}$ & $3.0 \pm 0.4^{\mathrm{a}}$ & $2.9 \pm 0.3^{\mathrm{a}}$ & $1.4 \pm 0.1^{\mathrm{b}}$ \\
\hline
\end{tabular}

Notes. Values are mRNA levels relative to control. Mean \pm SEM. $n=8$ per group.

Values with a different superscript for each gene are significantly different $(\mathrm{P}<0.05)$.

Table 3: Hepatic Protein Levels of Mice Fed AIN93G/M Diet Supplemented with BGA for 6 Months.

\begin{tabular}{|l|c|c|c|c|}
\hline & Control & NO 2.5\% & No 5\% & SP 2.5\% \\
\hline mSREBP-2 & $1.0 \pm 0.1$ & $1.3 \pm 0.1$ & $0.9 \pm 0.1$ & $1.4 \pm 0.2$ \\
\hline LDLR & $1.0 \pm 0.1^{\mathrm{b}}$ & $1.5 \pm 0.2^{\mathrm{a}}$ & $1.0 \pm 0.1^{\mathrm{b}}$ & $1.3 \pm 0.1^{\mathrm{ab}}$ \\
\hline FAS & $0.8 \pm 0.2^{\mathrm{b}}$ & $1.6 \pm 0.2^{\mathrm{ab}}$ & $2.2 \pm 0.5^{\mathrm{a}}$ & $1.7 \pm 0.1^{\mathrm{a}}$ \\
\hline
\end{tabular}

Notes: Values are arbitrary unit. Mean \pm SEM. $n=8$ per group.

Values with a different superscript for each protein are significantly different $(\mathrm{P}<0.05)$. 
the endoplasmic reticulum via sterol-induced interaction of SCAP with INSIGs $[33,34]$. When cellular cholesterol is low, disassociation of SCAP from INSIGs frees SCAP/SREBP-2 to the Golgi where mature SREBP-2 is released by site-1-protease and site-2-protease-mediated two step proteolytic cleavages $[35,36]$. The mature SREBP-2 then enters the nucleus to induce transcription of its target genes. Despite significant increases in hepatic TC levels in mice, which were fed NO and SP, mature SREBP-2 protein and LDLR mRNA levels were not significantly different between control and the BGA-fed groups. It is likely that the increased level of TC in the liver by BGA supplementation may not reach threshold to inhibit maturation of SREBP-2. In our previous study, we observed increased expression of hepatic HMGR and LDLR by 5\% NO feeding in mice after 4 weeks [19]. As there was significant decrease in plasma TC by $5 \% \mathrm{NO}$ at 3 month of feeding in the present study, there might be an increase in hepatic LDLR expression at the early months. It is important to note that mice used in this study were not challenged by diet to induce hyperlipidemic conditions. Future study needs to be warranted to address if BGA can prevent hyperlipidemia induced by high fat and high cholesterol.

High circulating levels of TG level are a predictor of atherosclerosis [37]. In this study, we observed decreases in plasma TG concentrations in 5\% and 2.5\% NO group at 2 and 6 month of feeding while SP $2.5 \%$ significantly decreased TG levels at 4 and 6 months. The decreased plasma TG value at 2 months by $5 \%$ NO was consistent with our previous short-term in vivo study [19]. SREBP-1c is a transcription factor that regulates the expression of lipogenic genes including FAS and SCD-1 [38]. After 6 months of BGA supplementation, NO both at 2.5\% and $5 \%$ significantly increased two lipogenic genes such as ACC-1 and SCD-1 compared with control and SP groups. This may explain no significant difference in hepatic TG levels between control and NO groups. In contrast, SP did not increase the expression of lipogenic genes whereas there was a trend toward increase in genes for fatty acid $\beta$-oxidation. Therefore, marked reduction in hepatic TG levels by both $2.5 \%$ and $5 \%$ SP supplementation is likely due to elevated fatty acid $\beta$-oxidation. Studies have suggested that SP may protect against the development of nonalcoholic fatty liver disease (NAFLD) [12]. Future study should be warranted to evaluate a potential role of SP in the prevention of age-related liver diseases.

In summary, long-term supplementation of NO and SP provide lipid-lowering effects via the modulation of genes involved in cholesterol and lipid metabolism in the livers of mice. As we also previously reported that the algae did not exert adverse side-effect, NO and SP may be potentially used for preventing dyslipidemia-associated chronic diseases.

\section{Conclusions}

In conclusion, our current study suggests that long-term consumption of NO and SP supplementation improved lipid profile in the liver and plasma of mice that were fed a regular chow diet for 6 months. Although more studies are necessary, together with our previous report on the safety of NO and SP consumption [21], this study supports that the BGA species may be developed as a natural product for the prevention of CVD and NAFLD.

\section{Acknowledgements}

This work was supported by National Institute Health grant R21AT005152 and fund from College of Agriculture and Natural Resources at the University of Connecticut to J. Lee.

\section{References}

1. Lusis AJ (2000) Atherosclerosis. Nature 407(6801): 233-241.

2. Chambless LE, Heiss G, Folsom AR, Rosamond W, Szklo M, et al. (1997) Association of coronary heart disease incidence with carotid arterial wall thickness and major risk factors: the Atherosclerosis Risk in Communities (ARIC) Study, 1987-1993. Am J Epidemiol 146(6): 483494.

3. Silva MA, Swanson AC, Gandhi PJ, Tataronis GR (2006) Statin-related adverse events: a meta-analysis. Clin Ther 28(1): 26-35.

4. Thompson PD, Clarkson P, Karas RH (2003) Statin-associated myopathy. JAMA 289(13): 1681-1690.

5. Olson JM (2006) Photosynthesis in the Archean era. Photosynth Res 88(2): 109-117.

6. Ciferri O (1983) Spirulina, the edible microorganism. Microbiol Rev 47(4): 551-578.

7. Parikh P, Mani U, Iyer U (2001) Role of Spirulina in the Control of Glycemia and Lipidemia in Type 2 Diabetes Mellitus. J Med Food 4(4): 193-199.

8. Regunathan C, Wesley SG (2006) Pigment deficiency correction in shrimp broodstock using Spirulina as a carotenoid source. Aquaculture Nutrition 12(6): 425-432.

9. Singh S, Kate BN, Banerjee UC (2005) Bioactive compounds from cyanobacteria and microalgae: an overview. Critical Reviews in Biotechnology 25(3): 73-95.

10. Qiu B, Liu J, Liu Z, Liu S (2002) Distribution and ecology of the edible cyanobacterium Ge-Xian-Mi (Nostoc) in rice fields of Hefeng County in China. Journal of Applied Phycology 14(5): 423-429.

11.Schaeffer DJ, Malpas PB, Barton LL (1999) Risk assessment of microcystin in dietary Aphanizomenon flos-aquae. Ecotoxicol Environ Saf 44(1), 73-80.

12. Ku CS, Yang Y, Park Y, Lee J (2013) Health benefits of blue-green algae: prevention of cardiovascular disease and nonalcoholic fatty liver disease. J Med Food 16(2): 103-111.

13. Rodriguez-Hernandez A, Ble-Castillo JL, Juarez-Oropeza MA, DiazZagoya JC (2001) Spirulina maxima prevents fatty liver formation in CD-1 male and female mice with experimental diabetes. Life Sci 69(9): 1029-1037.

14. Samuels R, Mani UV, Iyer UM, Nayak US (2002) Hypocholesterolemic effect of spirulina in patients with hyperlipidemic nephrotic syndrome. J Med Food 5(2): 91-96.

15.Esser MT, Mori T, Mondor I, Sattentau QJ, Dey B, et al. (1999) Cyanovirin-N binds to gp120 to interfere with CD4-dependent human immunodeficiency virus type 1 virion binding, fusion, and infectivity but does not affect the CD4 binding site on gp120 or soluble CD4induced conformational changes in gp120. J Virol 73(5): 4360-4371.

16. Knubel G, Larsen LK, Moore RE, Levine IA, Patterson GM (1990) Cytotoxic, antiviral indolocarbazoles from a blue-green alga belonging 
to the Nostocaceae. J Antibiot (Tokyo) 43(10): 1236-1239.

17. Smith CD, ZhangX, Mooberry SL, Patterson GM, Moore RE (1994) Cryptophycin: a new antimicrotubule agent active against drugresistant cells. Cancer Res 54(14): 3779-3784

18. Hori K, Ishibashi G, Okita T (1994) Hypocholesterolemic effect of bluegreen alga, ishikurage (Nostoc commune) in rats fed atherogenic diet Plant Foods Hum Nutr 45(1): 63-70.

19. Rasmussen HE, Blobaum KR, Jesch ED, Ku CS, Park YK, et al. (2009) Hypocholesterolemic effect of Nostoc commune var. sphaeroides Kutzing, an edible blue-green alga. Eur J Nutr 48(7): 387-394.

20. Rasmussen HE, Blobaum KR, Park YK, Ehlers SJ, Lu F, et al. (2008) Lipid extract of Nostoc commune var. sphaeroides Kutzing, a bluegreen alga, inhibits the activation of sterol regulatory element binding proteins in HepG2 cells. J Nutr 138(3): 476-481.

21. Yang Y, Park Y, Cassada DA, Snow DD, Rogers DG, et al. (2011) In vitro and in vivo safety assessment of edible blue-green algae, Nostoc commune var. sphaeroides Kützing and Spirulina plantensis. Food Chem Toxicol 49(7): 1560-1564.

22. Park YK, Rasmussen HE, Ehler SJ, Blobaum KR, Lu F, et al. (2008) Repression of proinflammatory gene expression by lipid extract of Nostoc commune var sphaeroides Kutzing, a blue-green alga, via inhibition of nuclear factor-kappa B in RAW 264.7 macrophages. Nutr Res 28(2): 83-91.

23. Betteridge DJ (2011) Lipid control in patients with diabetes mellitus. Nat Rev Cardiol 8(5): 278-290

24. Lloyd-Jones D, Adams RJ, Brown TM, Carnethon M, Dai S, et al. (2010) Heart disease and stroke statistics--2010 update: a report from the American Heart Association. Circulation 121(7): e46-e215.

25. Cheong SH, Kim MY, Sok DE, Hwang SY, Kim JH, et al. (2010) Spirulina prevents atherosclerosis by reducing hypercholesterolemia in rabbits fed a high-cholesterol diet. J Nutr Sci Vitaminol (Tokyo) 56(1): 34-40.

26. Iwata K, Inayama T, Kato T (1990) Effects of Spirulina platensis on plasma lipoprotein lipase activity in fructose-induced hyperlipidemic rats. J Nutr Sci Vitaminol (Tokyo) 36(2), 165-171.

27. Lee EH, Park JE, Choi YJ, Huh KB, Kim WY (2008) A randomized study to establish the effects of spirulina in type 2 diabetes mellitus patients. Nutr Res Pract 2(4): 295-300.

28. Briggs MR, Yokoyama C, Wang X, Brown MS, Goldstein JL (1993)
Nuclear protein that binds sterol regulatory element of low density lipoprotein receptor promoter. I. Identification of the protein and delineation of its target nucleotide sequence. J Biol Chem 268(19): 14490-14496.

29. Ericsson J, Jackson SM, Lee BC, Edwards PA (1996) Sterol regulatory element binding protein binds to a cis element in the promoter of the farnesyl diphosphate synthase gene. Proc Natl Acad Sci U S A 93(2): 945-950.

30. Guan G, Jiang G, Koch RL, Shechter I (1995) Molecular cloning and functional analysis of the promoter of the human squalene synthase gene. J Biol Chem 270(37): 21958-21965.

31. Osborne TF (1995) Transcriptional control mechanisms in the regulation of cholesterol balance. Crit Rev Eukaryot Gene Expr 5(3-4): 317-335.

32. Wang X, Briggs MR, Hua X, Yokoyama C, Goldstein JL, et al. (1993) Nuclear protein that binds sterol regulatory element of low density lipoprotein receptor promoter. II. Purification and characterization. J Biol Chem 268(19): 14497-14504.

33. Yabe D, Brown MS, Goldstein JL (2002) Insig-2, a second endoplasmic reticulum protein that binds SCAP and blocks export of sterol regulatory element-binding proteins. Proc Natl Acad Sci U S A 99(20), 12753-12758.

34. Yang T, Espenshade PJ, Wright ME, Yabe D, Gong Y, et al. (2002) Crucial step in cholesterol homeostasis: sterols promote binding of SCAP to INSIG-1, a membrane protein that facilitates retention of SREBPs in ER. Cell 110(4), 489-500.

35. Aagaard-Tillery KM, Grove K, Bishop J, Ke X, Fu Q, et al. (2008) Developmental origins of disease and determinants of chromatin structure: maternal diet modifies the primate fetal epigenome. J Mol Endocrinol 41(2): 91-102.

36. Goldstein JL, Rawson RB, Brown MS (2002) Mutant mammalian cells as tools to delineate the sterol regulatory element-binding protein pathway for feedback regulation of lipid synthesis. Arch Biochem Biophys, 397(2): 139-148.

37. Talayero, BG, Sacks FM (2011) The role of triglycerides in atherosclerosis. Curr Cardiol Rep 13(6): 544-552.

38. Horton JD, Goldstein JL, Brown MS (2002) SREBPs: activators of the complete program of cholesterol and fatty acid synthesis in the liver. J Clin Invest 109(9): 1125-1131. 